like arrogance for Chandra to have been so determined to do the right thing, we have to recognize that, almost always, he is right.

Wali's account of Chandrasekhar's life is a social and intellectual education; it recalls another era, and a scientific style that is out of fashion (no fifty Physical Review Letters a year from Chandra). The only failure of the book is that it presumes a certain familiarity with Chandra's achievements. Readers may well want to know what Chandra did after the momentous work on white dwarfs and the battle with Eddington, but Wali describes an eminent, greatly admired scientist without sufficiently explaining the eminence and admiration. It would be a shame if this kept a large audience from the book: there is much to be enjoyed in it.

David Lindley is an Associate Editor of Nature.

Just published by Chicago University Press is volume 5 of the selected papers of $S$. Chandrasekhar: Relativistic Astrophysics. Price is £59.95, \$86.25 (hbk); £23.95, $\$ 34.50$ (pbk).

\section{Astro aesthetics}

\section{David W. Hughes}

Universe. By Michael Rowan-Robinson. Longman: 1990. Pp.180. £17.95. Published in the United States by Freeman as Our Universe: An Armchair Guide. $\$ 24.95$.

ASTRONOMERS usually, and quite understandably, wince when confronted with the following quotation.

When I heard the learn'd astronomer,

When the proofs, the figures, were ranged in columns before me,

When I was shown the charts and diagrams, to add, divide and measure them,

When I sitting heard the astronomer where he lectures with much applause in the lecture room,

How soon unaccountable I became tired and sick,

Till rising and gliding out I wander'd off by myself,

In the mystical moist night-air, and from time to time,

Look'd up in perfect silence at the stars.

(W. Whitman, "By the Roadside".)

Rowan-Robinson uses this quotation to conclude the preface of Universe. The quotation is redolent with hints of C. P. Snow's two cultures. One culture is supposedly standing in awe and wonder and is receptive to beauty, and the other culture, replete with instruments, graph paper and computers, is only happy when it measures, dissects and understands. It is easy to see which side Whitman is on. He is liberal with his artistic sneers. To Whit-

man, the scientist is a deflowerer as well as a philistine. Whitman clearly knew too few. The joy of reading Universe was also not his.

Rowan-Robinson has taken great pains, not only to stride over the cultural divide, but also to pull the two sides together. His underlying thesis is that ignorance is not bliss; beauty is enhanced by understanding, and the more you know, the more the joy increases.

Universe is divided into twenty chapters, each devoted to a single object. Comets rub shoulders with the nearest star to the Sun, Vega with Mira, the ring nebula with the Crab, Andromeda with the Magellanic Clouds, radio galaxies with quasars and the Starburst galaxy with the Virgo cluster. The selection favours the rare, the exotic and the spectacular. New stars, demon stars, variability, explosions, gas jets and death throes are in, the mundane - the normal, slow, astrophysical drudgery of ordinary stars and planets - is out. In fact all the planets are out. Something as small, rocky and wet as the Earth is merely regarded as a punch bag for incoming asteroidal fragments and a supporting surface for astronomical observatories. Our life and the possibility of life elsewhere is not considered. The only thing that Rowan-Robinson seems to find really exciting within the nearest four light Shooting stars - Historical woodcut of the Leonids meteor years is our once-in-a-lifetime shower, reproduced from Universe. encounter with Halley's Comet. But this fact underlines one of the joys of the book. It is a personal selection and a personal romp through the visual heavenly wonders. I should stress the word 'visual'. The ideas that underlie the origin and evolution of the Universe and underlie our ever-changing perception of what the Universe contains, feature much less in this book than those contents of the Universe that can be easily imaged.

Each of the twenty objects is dealt with in nine pages. To say that the book is well illustrated is an understatement. It is a visual delight and colour abounds. Even Whitman would appreciate the astronomical aesthetic enhancement that has resulted from the employment of large telescopes and spacecraft instrumentation. Even he would realize that standing moistly and gazing silently is a poor substitute for the more modern endeavours of the astronomical community.

The two cultures still exist. They still sneer and snipe at each other from their close-guarded battlements. But bridges can be built and astronomers have an easier task than the majority of scientists.

Rowan-Robinson has produced a beautiful, stimulating and rewarding book. It is a feast for both the mind and the eye. The scientist is treated to a host of literary quotations and artistic reproductions, and the liberal artist to a careful exposition of the physical and chemical mechanisms that underpin the activities of the exotic bodies in the Universe. There is something in this book for us all.

David W. Hughes is Reader in Astronomy in the Department of Physics, University of Sheffield, S3 7RH, UK 